\title{
Heat transfer in a 180 deg turn channel
}

\author{
by G. Cardone, T. Astarita and G.M. Carlomagno
}

University of Naples - DETEC, P.le Tecchio, $80-80125$ Naples, ITALY, Tel. ++39817682178; Fax ++39 812390364

\begin{abstract}
The aims of the present study are to obtain detailed surface flow visualization and convective heat transfer measurements nearby a $180 \mathrm{deg}$ sharp turn in a square channel, and to prove that the use of infrared thermography may be appropriate to experimentally study this type of problems. Surface flow visualization and heat transfer measurements are performed by means of the heated-thin-foil technique and results are presented in terms of temperature maps and Nusselt number $\mathrm{Nu}$ distributions. $\mathrm{Nu}$ is computed by means of the local bulk temperature. The Reynolds number, based on average inlet velocity and hydraulic diameter of the channel, is varied between $1.6 \times 10^{4}$ and $5.5 \times 10^{4}$.
\end{abstract}

\section{Introduction}

The thermal efficiency of gas turbine engines strongly depends on the gas entry temperature, the higher the latter, the more efficient the turbine thermal cycle. Present advanced gas turbines operate at gas entry temperatures much higher than metal creeping temperatures and therefore require intensive cooling of their blades especially in the early stages. A classical way to cool turbine blades is by internal forced convection: generally, cooling air from the compressor is supplied through the hub section into the blade interior and, after flowing through a serpentine passage, is discharged at the blade trailing edge. The serpentine passage is mostly made of several adjacent straight ducts, spanwise aligned, which are connected by $180 \mathrm{deg}$ turns. The presence of these turns causes separation of the flow and so high variations of the convective heat transfer coefficients may occur with subsequent increased thermal stresses in the blade wall. The $180 \mathrm{deg}$ turn may be also encountered in other heat exchange devices.

Several works, both experimental and numerical, deal with the heat transfer aspects in rectangular channels with, more or less sharp, 180deg turns and with, or without, roughening ribs at the wall. Among others, the work of Metzger and Sahm [1], Fan and Metzger [2], Han et al. [3], Chandra et al. [4], Chyu [5], Wang and Chyu [6], Arts et al. [7], Lau et al. [8] is acknowledged. Metzger and co-workers report on a number of researches on regionallyaveraged heat transfer measurements for flow through rectangular channels with sharp $180 \mathrm{deg}$ turns. Chyu [5] also presents regionally-averaged heat transfer coefficients in two-pass and three-pass square channels measured by means of naphthalene sublimation.

Han et al. [3] and Chandra et al. [4] published a more detailed analysis about local heat transfer distributions along discrete lines, on smooth and rib-roughened walls, also obtained by means of the naphthalene sublimation technique.

Wang and Chyu [6] numerically solved with a finite difference method the three-dimensional transport equations and studied, for a given Reynolds number, the influence of the turning configuration on the convective heat transfer coefficient in a square duct with a $180 \mathrm{deg}$ turn.

Two investigations, which make use of the heated-thin-foil technique [9] and where the temperature is measured by means of thermochromic liquid crystals, have recently been published. The first one, carried out by Arts et al. [7], reports on Nusselt number distributions in 
a rectangular two-pass channel having an aspect ratio (width to height ratio) equal to 2 and a sharp 180deg turn. The second investigation, reported by Lau et al. [8], presents local heat transfer coefficients in four-pass channels, with different aspect ratios. These two works present the local heat transfer distribution around 180 deg turns. However, Arts et al. [7] do not specify any heating in the area underneath the partition wall, whereas Lau et al. [8] mention explicitly that this area is heated in their experiments. This causes overheating of the zone close to the partition wall and non-symmetric $\mathrm{Nu}$ distribution in the first and the fourth straight channels, even far away from the turns. Notwithstanding the conduction losses perpendicular to the measuring surface are considered in the two papers, tangential (along the wall) thermal conduction may affect significantly the real temperature distribution on the channel wall due to the relatively thick walls of the channel as compared to its width. Finally, in both experiments the electrical power input had to be varied to account for the limited range of liquid crystals with subsequent variations of the local bulk temperatures.

The need to produce detailed and reliable heat transfer distributions is not only important per se, but is also relevant to validate computer programs which are often used to study these complex flows. The main objective of the present work is to perform accurate measurements of the heat transfer distribution nearby a 180deg sharp turn in a square channel by means of infrared thermography. Another aim is to show that the use of infrared thermography in these type of problems may be advantageous on account of its relatively good spatial resolution and thermal sensitivity. Moreover, the use of thermography matches both qualitative and quantitative requirements [10]. The essential features of this methodology are [9]: it is nonintrusive; it allows a complete two-dimensional mapping of the surface to be tested; the video signal output may be treated by digital image processing.

\section{Experimental apparatus and procedure}

An open air driven flow channel, which consists of two adjacent straight ducts connected by a $180 \mathrm{deg}$ turn schematically shown in Figure 1 , is used. The length ahead of the $180 \mathrm{deg}$ turn, which is $2000 \mathrm{~mm}$, ensures an almost hydro-dynamically fully developed flow. Tolerances are \pm $0.5 \mathrm{~mm}$ on channel width and $\pm 0.2 \mathrm{~mm}$ on channel height. The central partition wall, which divides the two adjacent ducts, is $16 \mathrm{~mm}$ thick. The distance of the central partition edge from the short side of the channel is kept equal to the channel width. The tip of the partition wall has a square shape.



Fig .1. Experimental apparatus.

From the channel the air is aspirated, through a laminar flow meter, by a centrifugal blower which is powered by means of a variable speed electric motor. Two Pt100 RTD's measure the temperature at the entrance $T_{1}$ and at the outlet $T_{2}$ of the channel so as to monitor the overall temperature increase of air. The channel walls are manufactured from $20 \mathrm{~mm}$ thick soft wood (to have a low thermal conductivity) but, in the turn zone for a length of about $1200 \mathrm{~mm}$, the two side walls of the channel are manufactured by making use of printed circuit boards $(3$ of $400 \mathrm{~mm}$ 


\section{http://dx.doi.org/10.21611/qirt.1996.020}

apiece for each side). The printed circuits are designed so as to achieve a constant heat flux over the surface (except beneath the partition wall) by Joule effect. Their tracks are $17 \mu \mathrm{m}$ thick, $3 \mathrm{~mm}$ wide and placed at $3.2 \mathrm{~mm}$ pitch; the overall thickness of the board is $0.52 \mathrm{~mm}$. A stabilized DC power source supplies the electric current to the circuit and the power input is monitored by precisely measuring voltage drop and current across it.

It is important to notice that, although the flow is almost fully developed from the fluid dynamic point of view, a thermal entry length has to develop at the beginning of the heated zone. The external (to channel) not thermally insulated surface of three printed circuits boards, which is viewed by the infrared camera, is coated with a thin layer of black paint which has an emissivity coefficient equal to about 0.95 in the wavelength of interest. The viewed surface is placed at the bottom of the channel and surrounded by black curtains so as to minimize losses due to natural convection as well as unknown reflected radiation from the environment.

The infrared camera measures the temperature map of the bottom wall which is correlated to the heat transfer coefficient by means of the heated-thin-foil steady state technique [3]. In particular, for each pixel of the digitized thermal image, the convective heat transfer coefficient $h$ is calculated as:

$$
h=\left(q_{w}-q_{r}-q_{\partial}\right) /\left(T_{w}-T_{b}\right)
$$

where $q_{w}$ is the Joule heat flux, $q_{r}$ the radiative flux to ambient and channel, $q_{c}$ the losses due to natural convection and to tangential conduction within the wall, $T_{w}$ and $T_{b}$ are the wall temperature and the local bulk temperature, respectively. Because of the low value of the pertinent Biot number, the heated wall may be considered isotherm across its thickness.

The radiative thermal losses $q_{r}$ are computed from the measured $T_{w}$, those due to natural convection are measured and the losses due to tangential conduction are also computed by means of $T_{w}$ maps. The local bulk temperature $T_{b}$ is evaluated by measuring the stagnation temperature $T_{1}$ at the channel entrance and by making a one-dimensional energy balance along the channel, i.e. along the channel main axis; triangular heating sections are considered in the turning zone. By measuring $T_{1}, T_{2}$ and the air mass flow rate for each test run, an overall energy balance is also performed so as to compare the energy received by the fluid with the net electric power input.

The heat transfer coefficients are computed in non-dimensional form by means of the local Nusselt number:

$$
N u=h D / \lambda
$$

where $D$ is the hydraulic diameter of the channel and $\lambda$ the thermal conductivity coefficient of air evaluated at film temperature.

Tests are carried out for Reynolds number $R e$ values ranging from $1.6 \times 10^{4}$ to $5.5 \times 10^{4}$. Re is defined in the conventional way:

$$
R e=V D / v
$$

where $V$ and $v$ are the average inlet velocity in the channel and the kinematic viscosity coefficient of air, respectively.

The infrared thermographic system employed is the AGEMA Thermovision 900 . The field of view (which depends on the optics focal length and on the viewing distance) is scanned by the $\mathrm{Hg}$-Cd-Te detector in the 8-12 $\mu \mathrm{m}$ infrared window. Nominal sensitivity, expressed in terms of noise equivalent temperature difference, is $0.07^{\circ} \mathrm{C}$ when the scanned object is at ambient temperature. The scanner spatial resolution is 235 instantaneous fields of view per line at $50 \%$ 
slit response function. A $10^{\circ} \times 20^{\circ}$ lens is used, during the tests, at a viewing distance of $2.1 \mathrm{~m}$ which gives a field of view of about $0.24 \times 0.48 \mathrm{~m}^{2}$ i.e. approximately one third of the heated zone; this is done so as to obtain a higher spatial resolution in the thermal image. Therefore, to measure temperatures on the whole heated zone, three different thermal images are taken and patched up. Each image is digitised in a frame of $136 \times 272$ pixels at 12 bits. An application software can perform on each thermal image: noise reduction by numerical filtering; computation of temperature and heat transfer correlations.

\section{Results and discussion}

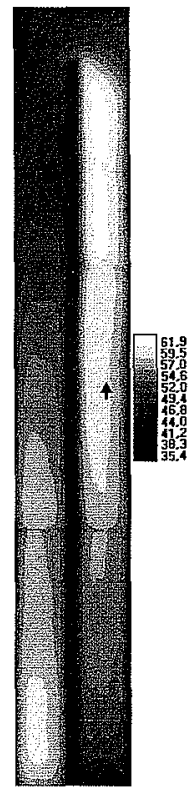

Fig. 2.

In order to appreciate the efficacy of thermography to perform this type of surface flow visualizations, the thermogram of Figure 2 shows the temperature map of the heated wall for the following testing conditions: $T_{1}=23^{\circ} \mathrm{C}, q_{w}=1185$ $W / m^{2}, R e=32,000$. As the scale on the right side of the thermogram shows, each gray level represents a temperature band. White and black zones indicate temperatures above $61.9^{\circ} \mathrm{C}$ and below $35.4^{\circ} \mathrm{C}$, respectively.

The temperature map of Figure 2 is obtained by joining three different thermal images acquired for the same testing conditions; the patching lines between two boards are visible. It has to be pointed out that, apart from the thermal losses and the continuous increase of the bulk temperature $T_{b}$ along the channel. (which, in the present tests, is of the order of few degrees Celsius), since the heat flux is practically constant over the surface, the higher the wall temperature $T_{w}$, the lower the convective heat transfer coefficient $h$ and viceversa. Furthermore, it has to be stressed that temperature data are not reliable in the neighborhood of walls since the latter, which are bonded to the printed circuit board and are not heated, tend to behave like fins in a stream and, therefore, produce in their vicinity a wall temperature decrease owing to some thermal conduction within the board. In addition, it has to be highlighted that iso-temperature contours may be wavy, or discontinuous at the printed circuit junction lines, in regions where the temperature is relatively constant. This because of the high sensitivity of the infrared camera to very small changes in the testing conditions between acquisition of two subsequent thermal images and mainly because of a slightly lower wall heat flux in the bottom circuit. In fact, this circuit has an electrical resistance $8 \%$ lower than that of the other two and all circuits are connected in series.

By moving streamwise along the channel, the quasi-regular trend of the temperature distribution across the first duct, at the beginning of the heated zone, proves a regular behavior of the flow. The rapid increase of the wall temperature in this part of the channel accounts for the developing thermal boundary layer which, of course, starts at the starting of the heated zone. Two high heat transfer regions are observed downstream of first and second corners of the outer wall which are due to the "jet" effect of the flow through the bend. This effect has also been revealed by previous authors [5,7]. Ahead of the exit of the heated zone a completely redeveloped flow is practically recovered.

The distribution of the local Nusselt number $N u$, normalized by its fully developed counterpart $\mathrm{Nu}^{\circ}$ in a straight duct without turn, i.e. the Dittus-Boelter correlation [11]:

$$
N u^{*}=0.024 \mathrm{Pr}^{0.4} \mathrm{Re}^{0.8}
$$

(Pr represents the Prandtl number), is shown in the maps of Figure. 3 for three different Reynolds numbers. The maps on the left side are relative to the heating from one side wall only while those on the right side refer to the heating from both side walls. 


\section{http://dx.doi.org/10.21611/qirt.1996.020}

The general trend in all maps is a Nusselt number, ahead of the turn, which practically coincides with $\mathrm{Nu}$. Across the turn a large increase in $\mathrm{Nu}$ occurs followed by a subsequent progressive decrease further downstream. Some very high heat transfer coefficient regions are present at the wall ; the first one is located on the end wall practically in front of the duct which enters the turn, slightly moved towards the partition wall axis; the second one, which exhibits the highest values of Nusselt number is just downstream of the second outer corner; the last one is near the partition wall about two diameters downstream of the second inner corner.

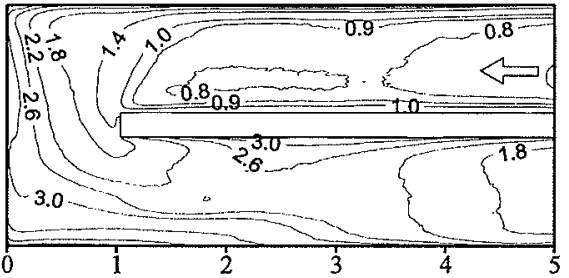

$R e=16000 ;$ one side heating

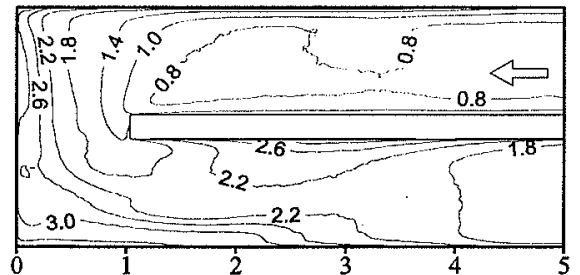

$\operatorname{Re}=30000 ;$ one side heating

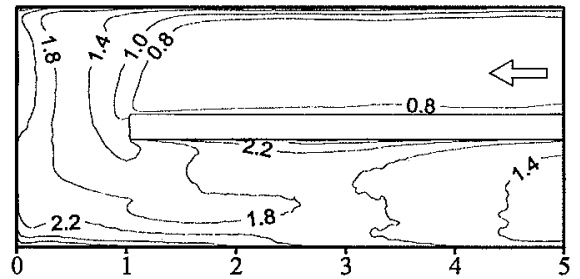

$R e=60000 ;$ one side heating

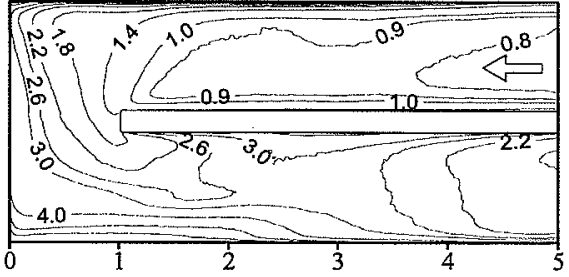

$R e=16000 ;$ two sides heating

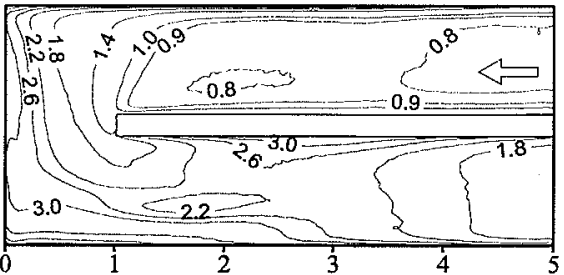

$R e=30000 ;$ two sides heating

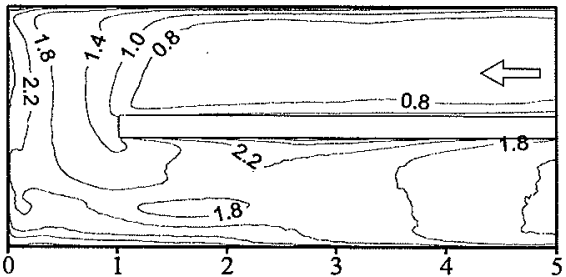

$R e=60000 ;$ two sides heating

Fig. 3 . Distribution of the local Nusselt number normalized by the value for fully developed flow.

Just before the first outer corner and attached to the external wall, a recirculation zone seems to appear (at the centre of which the Nusselt number attains a local minimum), but this minimum is always much higher than the corresponding value of $\mathrm{Nu}$. Another region of lower $N u$ is attached to the tip of the partition wall, slipping downstream, but $N u$ values are also in this case higher than the corresponding $\mathrm{Nu}^{*}$ ones. These two findings are much different from those found by Astarita et al. [12], who measured Nusselt numbers much lower than $N{ }^{*}$ in these two regions for a channel of aspect ratio equal to 5 . Ahead of the turn, data relative to one side heating shows a Nusselt number slightly higher than those for two side heating, while the opposite is true across and after the turn.

The present Nusselt number maps look very much different from the ones computed by Wang and Chyu [6], for different Reynolds number and thickness of the partition wall, as well as those measured by Arts et al. [7] who, however, tested a channel having an aspect ratio equal to 2. They are also different from those measured by Ekkad and Han [13] for testing conditions much similar to the present ones; e. g., in ref. [13] the second high Nu region appears quite far downstream of the second outer corner. 


\section{Conclusions}

Results for the $180 \mathrm{deg}$ turn show that the Nusselt number, ahead of the turn, practically coincides with $\mathrm{Nu}$ i based on the Dittus-Boelter correlation. Across the turn a large increase of $\mathrm{Nu}$ occurs followed by a subsequent progressive decrease further downstream. Some regions of high heat transfer coefficient are present at the side walls; the first one is located on the end wall practically in front of the duct which enters the turn slightly moved towards the partition wall axis; the second region, which exhibits the highest values of Nusselt number, is just downstream of the second outer corner; the last one is near the partition wall about two diameters downstream of the second inner corner. Just before the first outer corner and attached to the external wall, a recirculation zone at the centre of which the Nusselt number attains a local minimum seems to appear; but this minimum is always much higher than the corresponding value of $\mathrm{Nu}$. Another region of lower $\mathrm{Nu}$ is attached to the tip of the partition wall, slipping downstream, but $\mathrm{Nu}$ values also in this case are higher than the corresponding $\mathrm{Nu}$ ones.

\section{REFERENCES}

[1] METZGER (D. E.) and SAHM (M. K.) - Heat Transfer Around Sharp 180-Deg Turns in Smooth Rectangular Channels, ASME Journal of Heat Transfer, Vol. 108, pp. 500-506, 1986.

[2] FAN (C. S.) and METZGER (D. E.) - Effects Of Channel Aspect Ratio On Heat Transfer In Rectangular Passages With Sharp 180-Deg Turns, ASME Paper No. 87-GT-113, 1987.

[3] HAN (J. C.), CHANDRA (P. R.) and LAU (S. C.) - Local HeatMass Transfer Distributions Around Sharp 180-Deg Turns in Two-Pass Smooth and Rib-Roughened channel, ASME Journal of Heat Transfer, Vol. 110, pp. 91-98, 1988.

[4] CHANDRA (P. R.), HAN (J. C.) and LAU (S. C.) - Effect of Rib Angle on Local HeatMass Transfer Distribution in a Two Pass Rib Roughened Channel, ASME Journal of Turbomachinery, Vol. 110, pp. 223$241,1988$.

[5] CHYU (M. K.) - Regional Heat transfer in Two-Pass and Three-Pass Passages with 180-Deg Sharp Turns, ASME Journal of Heat Transfer, Vol. 113, pp. 63-70, 1991.

[6] WANG (T. S.) and CHYU (M. K.) - Influence of Turning geometry on Convective transport in Square Duct with a 180- degree Sharp Turn, Proc. Int. Symp. on Heat Transfer in Turbomachinery, Athens, 1992.

[7] ARTS (T.), LAMBERT DE ROUVROIT (M.), RAU (G.) and ACTON (P.). - Aero-Thermal Investigation of the Flow Developing in a 180 Degree Turn Channel, Proc. Int. Symp. on Heat Transfer in Turbomachinery, Athens, 1992.

[8] LAU (S. C.), RUSSELL (L. M.), THURMAN (D. R.) and HIPPENSTEELE (S. A.) - Visualization of Local Heat Transfer in Serpentine Channels with Liquid Chrystals, Proc. V Int. Symp. on Transport Phenomena and Dynamics of Rotating Machinery, Vol. A, pp. 411-423, Kaanapaly, Hawaii, 1994.

[9] CARLOMAGNO (G. M.) and DE LUCA (L.) - Heat Transfer Measurements by means of Infrared Thermography, in Handbook of Flow Visualization, W.J. Yang ed., pp. 531-553 Hemisphere, 1989.

[10] CARLOMAGNO (G. M.) - Heat Transfer Measurements by Means of Infrared Thermography, in Measurement Techniques, Von Karman Institute for Fluid Mechanics Lect. Series 1993-05, 1-114, RhodeSaint-Genese, 1993.

[11] KAYS (W. M.), and CRAWFORD (M. E.) Convective Heat And Mass Transfer, p. 319, Mc Graw-Hill, New York, 1993.

[12] Astarita (T.), Cardone (G.) and Carlomagno (G.M.), Heat Transfer and Surface Flow Visualization Around a 180deg Turn in a Rectangular Channel in Heat Transfer in Turbulent Flows, Anand N.K. et al. Eds., ASME HTD - Vol. 318, pp. 161-168, 1995.

[13] EKKAD (S.V.) and HAN (J.C.), Local Heat Transfer Measurements Near a Sharp $180^{\circ}$ Turn of a Twopass Smooth Square Channel with a Transient Liquid Crystal Image Technique, Proc. $V$ Int. Symp. on Transport Phenomena and Dynamics of Rotating Machinery, Kaanapaly, Vol. A, pp. 701-716, 1994. 